\title{
A FINITE ELEMENT PENALIZED DIRECT FORCING METHOD TO TAKE INTO ACCOUNT INFINITELY THIN IMMERSED BOUNDARIES IN A DILATABLE FLOW
}

\author{
GEORIS BILLO $^{1 *}$, MICHEL BELLIARD ${ }^{1}$ AND PIERRE SAGAUT ${ }^{2}$ \\ ${ }^{1}$ French Alternative Energies and Atomic Energy Commission (CEA), DES, IRESNE, DER, SESI, \\ LEMS \\ Cadarache \\ F-13118 Saint-Paul-Lez-Durance, France \\ ${ }^{2}$ Aix-Marseille University, CNRS, Centale Marseille \\ 38 rue Joliot-Curie \\ 13451 Marseille, France \\ *Presenting and corresponding author, georis.billo@cea.fr
}

Key words: Immersed Boundary Method, Finite Elements Method, Thermalhydraulics, Computational Fluid Dynamics, Projection methods for solving Navier-Stokes equations

\begin{abstract}
In the framework of the new passive safety systems developed by the French Atomic Energy Commission (CEA) for the second and third generations of nuclear reactors, a numerical simulation tool capable of modeling thin inflow obstacles is needed [1]. Considering its future use in shape optimization and thermalhydraulics safety studies, the tool must be the fastest, the most accurate and the most robust possible.
\end{abstract}

The aforementioned context has lead to the Computational Fluid Dynamics (CFD) modeling we are currently developing. For now, it involves a projection scheme to solve the dilatable Navier-Stokes equations and, to take into account obstacles, an adaptation of the Penalized Direct Forcing (PDF) method [2] a technique whose characteristics inherit from both penalty [3] and Immersed Boundary Method (IBM) [4] - to a Finite Element (FE) formulation. This first modeling offers two variants : one in which the velocity imposed at the vicinity of an obstacle is constant and another in which it is linearly interpolated using properties of the considered immersed boundary (normal vector, barycenter, characteristic function) and the FE basis functions. The results obtained via those two variants, for laminar flow, are in good agreement with analytical and experimental data. However, when compared to each other, it appears that the interpolation of the velocity imposed at the vicinity of the immersed boundary increases the mesh convergence order - which is very interesting, in term of accuracy/computation time ratio.

Some enhancements of the tool are also considered, mainly related to turbulence modeling. Indeed, the interpolating process, instead of being linear, could follow a turbulent wall law. 


\section{INTRODUCTION}

The increasing performance and safety requirements for the third generation of nuclear reactors led to new research and development studies. In this context, new innovative passive safety systems are designed to prevent or soften potential accidental or incidental situations. Some of those systems involve thin rigid motionless fins to guide the flow ( $c f$. Figure 1.a). The pressure drop, a quantity of interest for higher scales (i.e. reactor design), induced by those devices is greatly influenced by the shape of the fins. Thus, in order to carry out shape optimization and safety studies, a fast and robust numerical tool, able to faithfully model the new passive safety systems, is needed. For more information about the background, the interested reader can refer to [1]. The aimed applications involve a subsonic compressible twophase flow in non-equilibrium state. As a first approach, the Homogeneous Equilibrium Model could be considered to preserve low computational times while taking into account two-phase aspects. However, for purpose of clarity, only one-phase Navier-Stokes equations are considered in this paper. From this point of view, projection schemes are often used to deal with those equations [5] at low Mach number. To take into account the effect of the complex geometry of the fins on the flow, while preserving simple grids and short computation times, an immersed boundary approach has been chosen : the Penalized Direct Forcing (PDF) method, developed by M. BELLiARD et al. [2] in a Finite Difference context, whose characteristics inherits from both penalty [3] and direct forcing [6] techniques. We adapted this method to a Galerkin Finite Element (FE) formulation in order to interpolate the velocity imposed by the immersed boundaries in its vicinity using the FE discrete basis. At last, the flow regime, together with the complex geometry of the devices, also indicate that turbulence will be a major phenomenon. However,

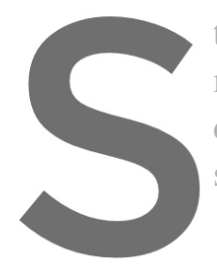

this immersed boundary

models, requiring a deep

document briefly describe

some simulation results

core barrel shell

(a)
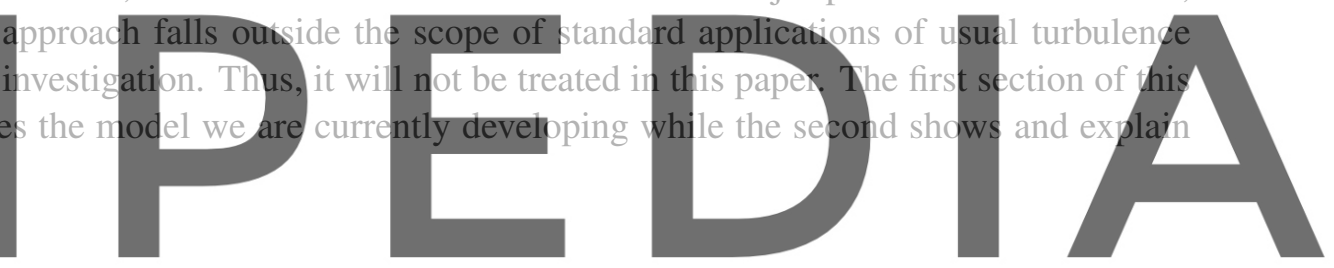

Register for free at https/pwwww.scipedia.com to download the velpsion without the watermark
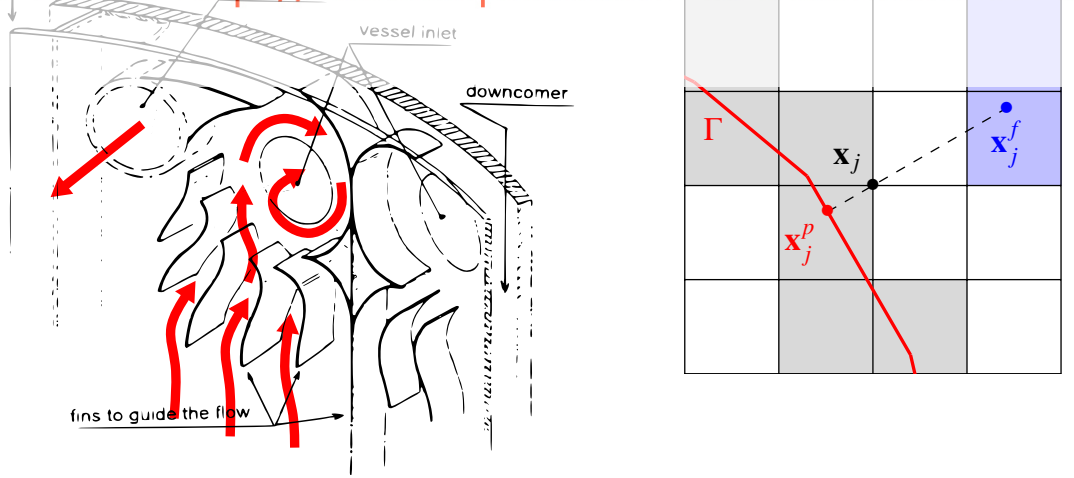

$\square: \chi_{e}=0$, "Purely fluid"

$\square: \chi_{e}=0$, "Not purely fluid"

$: \chi_{e}=1$

Figure 1: (a): Schematic representation of the flow limiter in an accidental situation. (b): Schematic representation of the interpolation stencil with $\mathbf{x}_{j}$ a grid node, $\mathbf{x}_{j}^{p}$ its approximate projection on the immersed obstacle $\Gamma$ and $\mathbf{x}_{j}^{f}$ a point which is not directly influenced by the immersed obstacle. 


\section{DESCRIPTION OF THE PROPOSED MODEL}

\subsection{Governing equations}

In the method we developed, the one-phase Navier-Stokes equations are considered:

$$
\begin{cases}\partial_{t}(\rho \mathbf{u})+\nabla \cdot(\rho \mathbf{u} \otimes \mathbf{u}-\overline{\bar{\sigma}}+\overline{\bar{I}} p)=\rho \mathbf{g} & \text { on } \Omega \\ \partial_{t}(\rho)+\nabla \cdot(\rho \mathbf{u})=0 & \text { on } \Omega \\ + \text { Boundary Conditions } & \text { on } \partial \Omega \\ + \text { Initial Conditions } & \text { on } \Omega\end{cases}
$$

with $\overline{\bar{I}} \in \mathbb{R}^{3 \times 3}$ the identity matrix, $\rho$ the fluid density, $p$ the pressure, $\mathbf{u}$ the fluid velocity, $\mathbf{g}$ the gravity vector and $\overline{\bar{\sigma}}$ the viscous stress tensor.

\subsection{Time discretization}

Our approach involves a quasi-implicit Euler scheme coupled with a three-step projection algorithm [5].

Those three steps are the following (with $\delta t$ the time step):

1. Prediction: only time, inertia, mechanical stress and source terms are considered. An intermediate velocity, called the predicted velocity and denoted $\mathbf{u}^{*}$, is computed:
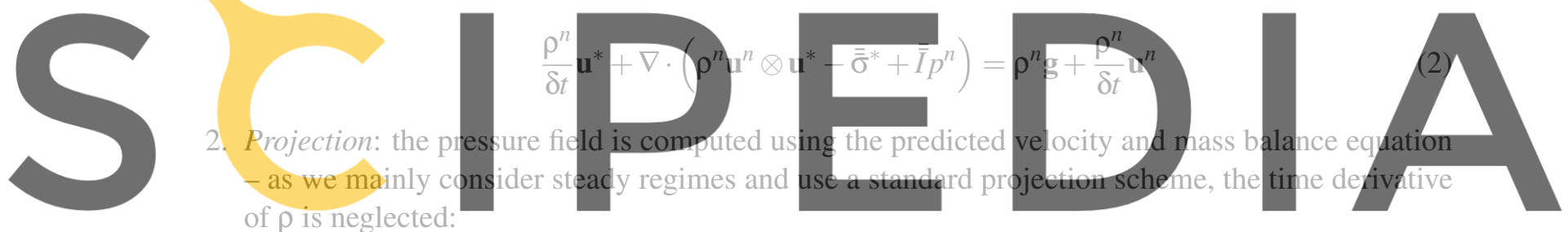

Register for free at https//www.scipedia,com to download the version without the watermark
\[ \Delta \phi^{n+1}=\frac{1}{\delta+} \nabla \cdot\left(\rho^{n} \mathbf{u}^{*}\right) \text { with } \phi^{n+1}=p^{n+1}-p^{n} \]

3. Correction: the velocity is computed using the pressure corrector gradient:

$$
\mathbf{u}^{n+1}=\mathbf{u}^{*}-\frac{\delta t}{\rho^{n}} \nabla \phi^{n+1}
$$

A Picard algorithm is actually implemented to make the advective term quasi-implicit. $\rho^{n}$ is computed using the variables taken at the previous step of the Picard algorithm to fill an equation of state.

\subsection{Penalized Direct Forcing method}

To take into account infinitely thin in-flow obstacles, denoted $\Gamma$, we use the PDF method. It inherits from the Immersed Boundary Method developed by C.S. PESKIN [7] (reviewed by J. MoHD-Yusof [6] in the Direct Forcing technique) because it consists in adding a new source term - which can be seen as a backmoving force - in the governing equations. However, it also involves a penalty parameter (i.e. a 
numerically infinite stiffness), inspired by the work of P. ANGOT et al. [3]. Finally, as shown by many ([8] and references within), this term, when used in a fractional-step algorithm, needs to be split between the prediction and projection equations to ensure consistency:

$$
\begin{aligned}
\frac{\rho^{n}}{\delta t}\left(\mathbf{u}^{*}-\mathbf{u}^{n}\right)+\nabla \cdot\left(\rho^{n} \mathbf{u}^{n} \otimes \mathbf{u}^{*}-\overline{\bar{\sigma}}^{*}+\overline{\bar{I}} p^{n}\right) & =\rho^{n} \mathbf{g}+\mathbf{f}_{1}^{n+1} \\
\frac{\rho^{n}}{\delta t}\left(\mathbf{u}^{n+1}-\mathbf{u}^{*}\right)+\nabla \phi^{n+1} & =\mathbf{f}_{2}^{n+1}
\end{aligned}
$$

with:

$$
\begin{aligned}
& \mathbf{f}_{1}^{n+1}:=\chi \frac{\rho^{n}}{\eta \delta t}\left(\mathbf{u}_{\Gamma}^{n+1}-\mathbf{u}^{*}\right) \\
& \mathbf{f}_{2}^{n+1}:=\chi \frac{\rho^{n}}{\eta \delta t}\left(\mathbf{u}^{*}-\mathbf{u}^{n+1}\right)
\end{aligned}
$$

where $0<\eta \ll 1$ is the penalty parameter, $\chi$ is the characteristic function of the obstacles and $\mathbf{u}_{\Gamma}^{n+1}$ is the velocity imposed by the obstacles (the value of the Dirichlet immersed boundary condition). When gathering terms together, we obtain:
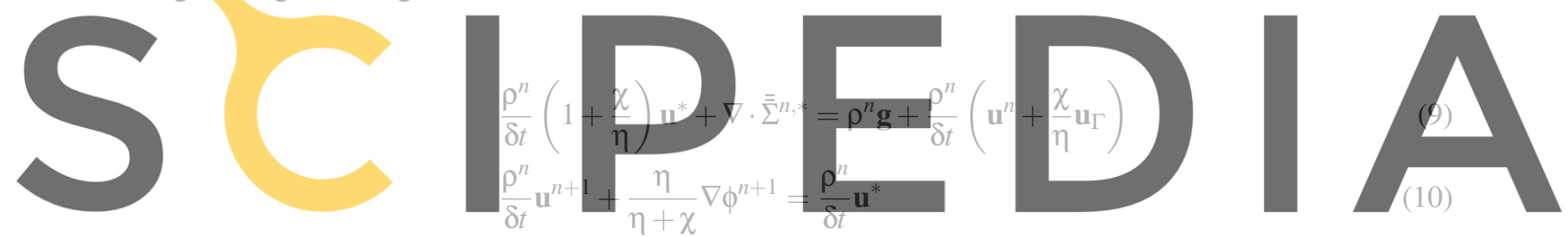

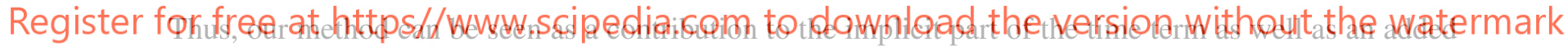

source term in the prediction equation and an added diffusivity in the projection equation. One can note

that those forcing terms are discontinuous among the immersed boundary $\Gamma$. This implies that the one associated to the projection equation has to be added before applying the divergence and substituting the mass equation - the actual substitution occurs at a discrete level.

\subsection{Finite Element discretization}

A Galerkin Finite Element Method (FEM) is used to achieve the space discretization of the problem [9]. This choice is motivated by the possibility of using the FE basis (potentially enriched) to interpolate the fluid velocity at the vicinity of an obstacle ( $c f$. following section) and, also, by the existence of a house-code [10] including immersed-boundary-like features. It is also worth noting that the general idea of the PDF method does not depend strongly on the space discretization.

In short, the computation domain is divided in hexahedral elements. For the velocity, a $\mathbb{Q}_{1}$ basis (i.e. trilinear decomposition at nodes) is used while, for pressure, a $\mathbb{Q}_{0}$ basis (i.e. the discrete pressure field is constant by element) is used. This pair of elements is known to be unstable but we kept it for two 
main reasons: it counts very few degrees of freedom per element (so it is quite fast) and the pressure instabilities are soften when the diffusivity is high enough - i.e. in laminar cases or when the turbulent viscosity is high, which is a case of interest for nuclear passive safety systems. The discrete version of the characteristic function of $\Gamma$ is denoted $\chi_{e}$ and is considered as $\mathbb{Q}_{0}$ (i.e. constant by element). If an element numbered $e$ is crossed by the immersed obstacle $\chi_{e}=1$, else $\chi_{e}=0$.

Using this pair of elements, we can write the discrete versions of equations (9) and (10) in an element numbered $e \in \mathbb{N}$ :

$$
\begin{aligned}
\left(\frac{1}{\delta t} \mathbf{M}_{e} \rho_{e}^{n}\left[1+\frac{\chi_{e}}{\eta}\right]-\mathbf{D}_{e} \mu_{e}^{n}+\mathbf{N}_{e} \rho_{e}^{n} \lambda^{n}\right) \lambda^{*} & =\frac{1}{\delta t} \mathbf{M}_{e} \rho_{e}^{n}\left(\lambda^{n}+\frac{\chi_{e}}{\eta} \lambda_{\Gamma}^{n+1}\right)+\mathbf{B}_{e} p_{e}^{n}+\mathbf{M}_{e} \rho_{e}^{n} \lambda_{g} \\
\mathbf{B}_{e}^{T} \mathbf{M}_{e}^{-1} \frac{\eta}{\eta+\chi_{e}} \mathbf{B}_{e} \phi_{e}^{n+1} & =-\frac{1}{\delta t} \mathbf{B}_{e}^{T} \rho_{e}^{n} \lambda^{*}
\end{aligned}
$$

with $\mathbf{M}_{e}$ (resp. $\mathbf{D}_{e}, \mathbf{N}_{e}$ and $\mathbf{B}_{e}$ ) the lumped mass [11] (resp. diffusivity, advective and gradient-divergence) elemental matrix, $\lambda^{n}$ (resp. $\lambda^{*}$ and $\lambda_{g}$ ) the components of the velocity (resp. predicted velocity and gravity vector) in the $\mathbb{Q}_{1}$ finite elements basis, $\phi_{e}^{n+1}$ (resp. $\rho_{e}^{n}$ ) the discrete pressure corrector (resp. fluid density) and, finally, $\lambda_{\Gamma}^{n+1}$ the decomposition of the imposed velocity $\mathbf{u}_{\Gamma}^{n+1}$ in the $\mathbb{Q}_{1}$ finite elements basis - those values are interpolated as explained in the following section.
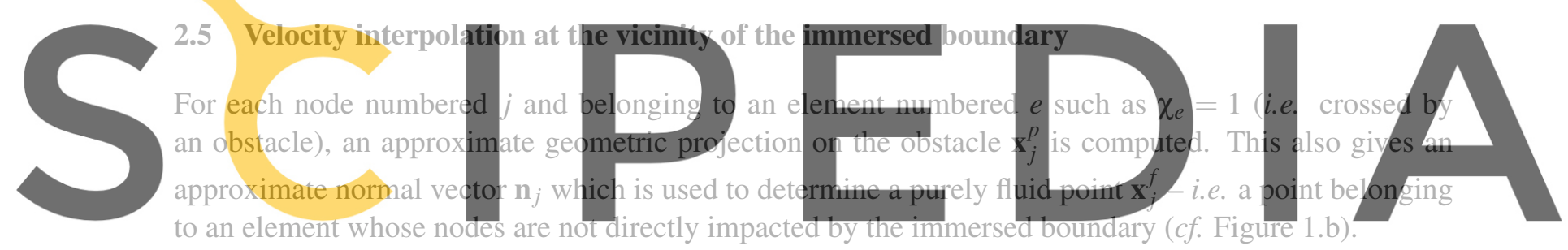

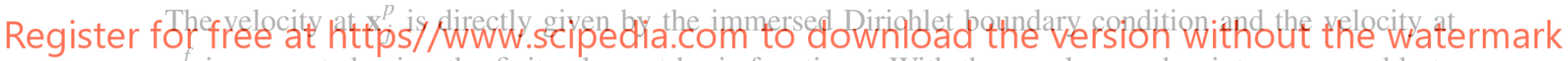

$\mathbf{x}_{j}^{f}$ is computed using the finite element basis functions. With those values and points, we are able to get an approximate value of the velocity at node $j$ by linear interpolation and to use it as the imposed velocity $\mathbf{u}_{\Gamma}$ presented in equation (5) - in this section, the notation of time indices is omitted for purpose of readability keeping in mind that this process occurs at each time step.

The velocity at $\mathbf{x}_{j}^{p}$, denoted $\mathbf{u}_{j}^{p}$, is directly given by the immersed Dirichlet boundary condition and the velocity at $\mathbf{x}_{j}^{f}$, denoted $\mathbf{u}_{j}^{f}$, is computed using the finite elements basis functions. With those value and points, we are able to create an approximate value of the velocity at node $j$ by linear interpolation and use it as the imposed velocity presented in equation (9):

$$
\mathbf{u}\left(\mathbf{x}=\mathbf{x}_{j}\right) \approx \mathbf{u}_{j}=\mathbf{u}_{j}^{p}+\frac{\mathbf{u}_{j}^{f}-\mathbf{u}_{j}^{p}}{\left|\mathbf{x}_{j}^{f}-\mathbf{x}_{j}^{p}\right|}\left|\mathbf{x}_{j}-\mathbf{x}_{j}^{p}\right|=\mathbf{u}_{\Gamma}
$$




\section{NUMERICAL RESULTS}

\subsection{Convergence order studies}

(a)
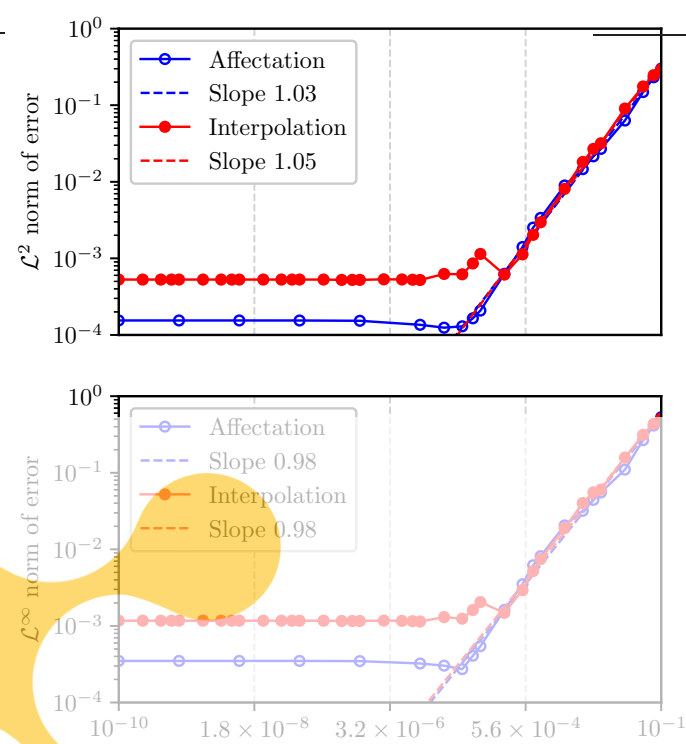

Figure 2: (a): the evolution with the mesh) and its analy (b): the evolution of the relat with respect to the grid step

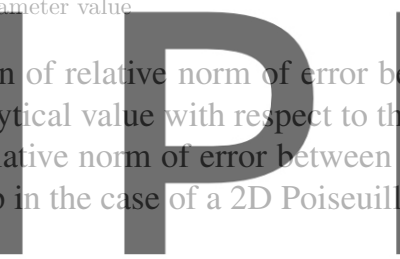

(b)
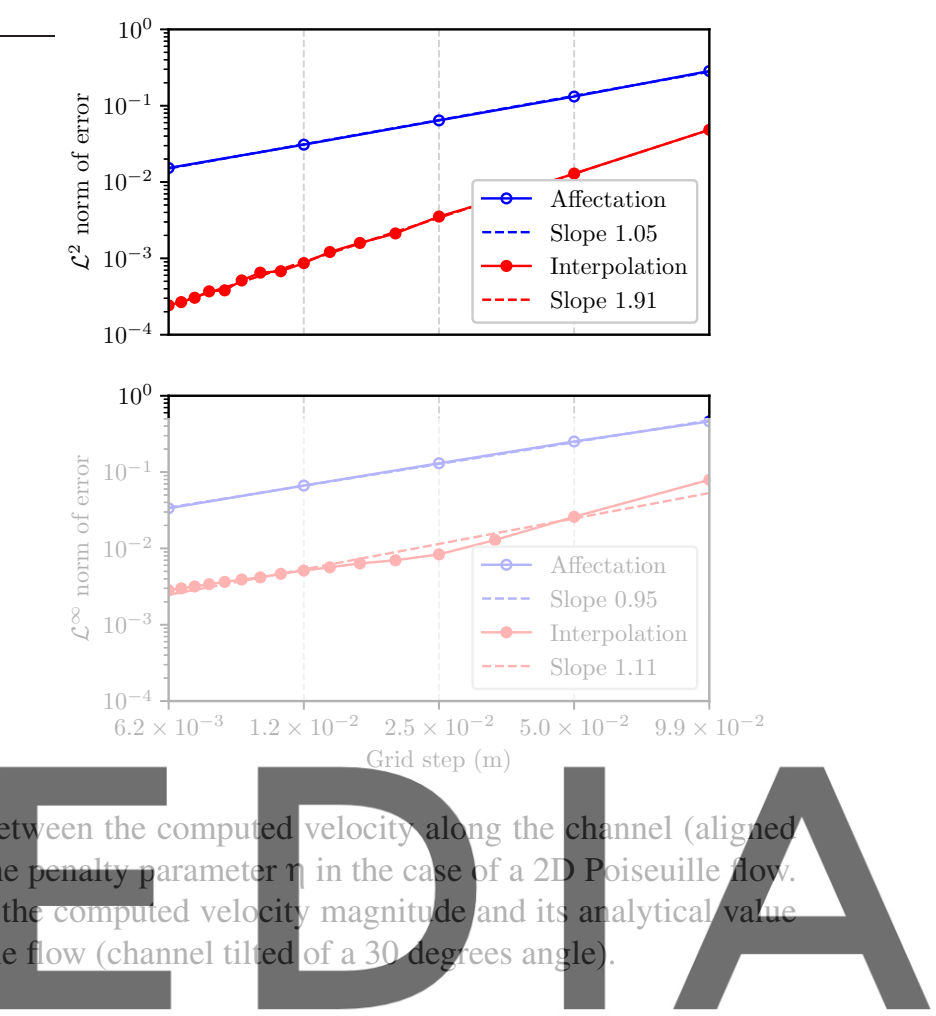

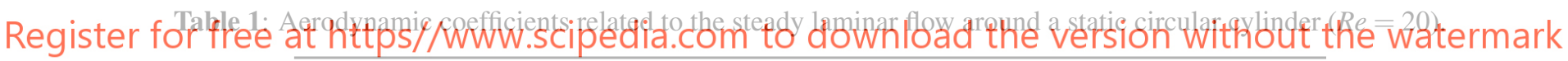

\begin{tabular}{|c|c|c|c|c|c|c|c|c|c|c|}
\hline & \multicolumn{2}{|l|}{$2 r / h$} & \multicolumn{7}{|c|}{ References } \\
\hline & & 10 & 20 & [12] & [13] & [14] & [15] & [16] & [17] & [18] \\
\hline \multirow[t]{2}{*}{$C_{d}$} & Ass. & 2.245 & 2.154 & 2.059 & 2.03 & 2.02 & 2.06 & 2.06 & 2.00 & 2.09 \\
\hline & Int. & 2.142 & 2.075 & 2.054 & & & & & & \\
\hline \multirow[t]{2}{*}{$L_{w}$} & Ass. & 1.297 & 1.166 & 0.925 & 0.92 & 0.90 & 0.94 & 0.93 & 0.91 & - \\
\hline & Int. & 0.749 & 0.901 & 0.900 & & & & & & \\
\hline
\end{tabular}

The behavior of our method with respect to the penalty parameter $\eta$ has been studied in the case of a $2 \mathrm{D}$ Poiseuille flow - the channel is aligned with the grid and the immersed boundary are conforming to the element faces in order to minimize the spatial error and focus on the penalty error. Figure 2.a) shows the $\mathcal{L}^{2}$ and $\mathcal{L}^{\infty}$ relative norms of error of the component of the velocity oriented along the channel obtained from several values of the penalty parameter. The blue curve corresponds to results obtained with a variant of the method in which the immersed Dirichlet boundary condition value is directly assigned to the imposed velocity $\mathbf{u}_{\Gamma}^{n+1}$. The red curve correspond to the results obtained by using the velocity 
Table 2: Aerodynamic coefficients related to the steady laminar flow around a rotating circular cylinder $(R e=20)$.

\begin{tabular}{|c|c|c|c|c|c|c|c|c|}
\hline & & \multicolumn{2}{|l|}{$2 r / h$} & \multicolumn{5}{|c|}{ References } \\
\hline & & 10 & 20 & [12] & [19] & [20] & {$[21]$} & {$[22]$} \\
\hline \multirow[t]{2}{*}{$C_{d}$} & Ass. & 2.057 & 1.984 & 1.8608 & 1.888 & 1.85 & 1.925 & 2.000 \\
\hline & Int. & 2.002 & 1.913 & 1.8679 & & & & \\
\hline \multirow[t]{2}{*}{$C_{l}$} & Ass. & 3.181 & 3.032 & 2.9419 & 2.629 & 2.75 & 2.617 & 2.740 \\
\hline & Int. & 2.970 & 2.868 & 2.7745 & & & & \\
\hline \multirow[t]{2}{*}{$\alpha\left(^{\circ}\right)$} & Ass. & 57.11 & 56.79 & 57.68 & 54.31 & 56 & 53.66 & 53.87 \\
\hline & Int. & 56.02 & 56.29 & 56.05 & & & & \\
\hline
\end{tabular}

Table 3: Aerodynamic coefficients related to the unsteady laminar flow around a static circular cylinder $(\operatorname{Re}=100)$.

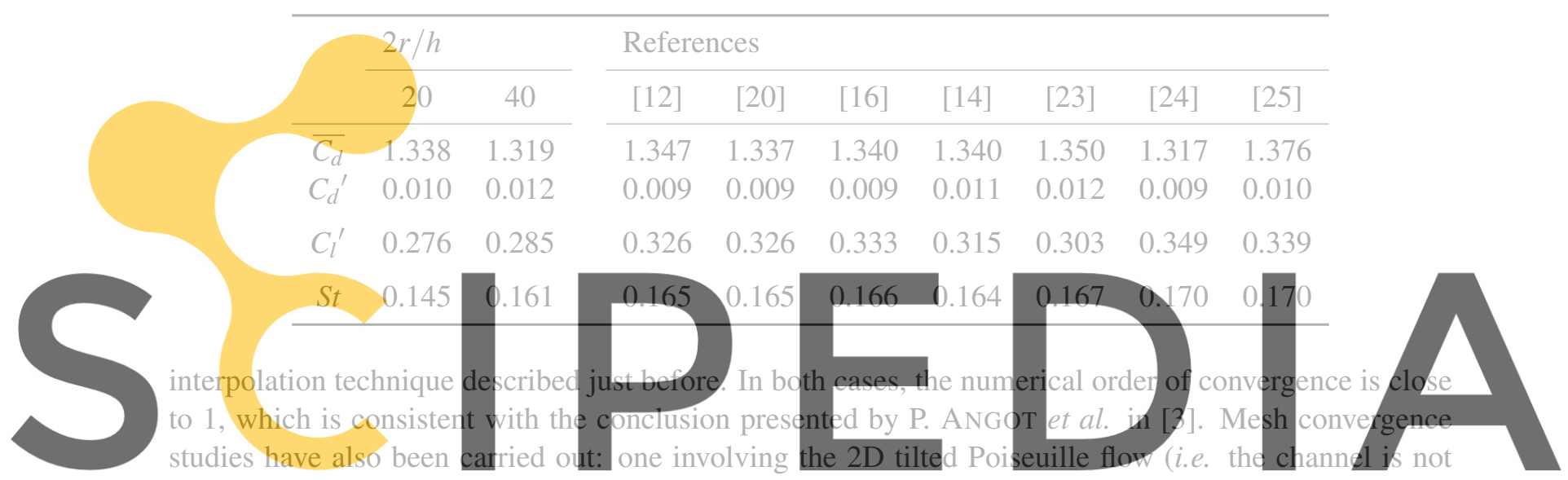

aligned with the mesh, it is tilted of an angle $\theta$ ), one involving the Taylor-Couette flow and, finally,

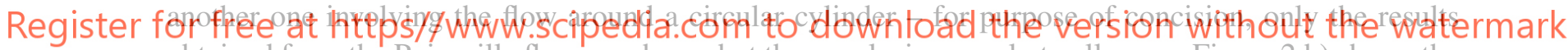
obtained from the Poiseuille flow are shown but the conclusions apply to all cases. Figure 2.b) shows the evolution of the relative norm of error with respect to the grid in the case of 2D Poiseuille flow with a channel tilted of a $30^{\circ}$ angle. The same variants of the methods are compared in terms $\mathcal{L}^{2}$ and $\mathcal{L}^{\infty}$ norm. As expected from the theory and the literature, a spatial order of convergence of almost 2 is reached when the imposed velocity is interpolated.

\subsection{Global quantities studies}

The flow around static and rotating circular cylinders have been studied at different Reynolds numbers - Figure 3 shows an example of the computed streamlines and isobars in the case of a static cylinder at Reynolds $R e=20$. Global quantities such as drag and lift coefficients $-C_{d}$ and $C_{l}$ with, in unsteady cases ( i.e. $R e=100$ ), their mean values denoted with a bar and their fluctuations denoted with an apostrophe. The angle between the aerodynamic force and the horizontal axis $(\alpha)$ and the Strouhal number (St) have been computed and compiled in Tables 1, 2, 3 and $4-r$ refers to the cylinder radius and $h$ to the space step so $2 r / h$ is an estimation of the number of elements included within the diameter of the cylinder. Those 
Table 4: Aerodynamic coefficients related to the unsteady laminar flow around a rotating circular cylinder $(R e=$ 100).

\begin{tabular}{|c|c|c|c|c|c|c|}
\hline & \multicolumn{2}{|l|}{$2 r / h$} & \multicolumn{4}{|c|}{ References } \\
\hline & 20 & 40 & [12] & [20] & [19] & [26] \\
\hline$\overline{C_{d}}$ & 1.143 & 1.097 & 1.12 & 1.1080 & 1.1890 & 1.0979 \\
\hline$C_{d}^{\prime}$ & 0.098 & 0.091 & 0.11 & 0.0986 & 0.1195 & 0.0988 \\
\hline$\overline{C_{l}}$ & 2.634 & 2.573 & 2.51 & 2.5040 & 2.4050 & 2.4833 \\
\hline$C_{l}^{\prime}$ & 0.285 & 0.291 & 0.37 & 0.3616 & 0.4427 & 0.3603 \\
\hline$S t$ & 0.151 & 0.165 & 0.165 & 0.1658 & 0.1732 & 0.1650 \\
\hline
\end{tabular}

Table 5: Aerodynamic coefficients related to the laminar flow past a NACA airfoil. Steady regimes are not recovered for lines colored in gray.

\begin{tabular}{|c|c|c|c|c|}
\hline \multirow[t]{2}{*}{$\theta$} & \multicolumn{2}{|l|}{$C_{d}$} & \multicolumn{2}{|l|}{$C_{l}$} \\
\hline & Interpolation & [27] & Interpolation & {$[27]$} \\
\hline 0 & 0.0563 & 0.0555 & 0.0094 & 0.0000 \\
\hline 1 & 0.0571 & 0.0559 & 0.0092 & 0.0184 \\
\hline
\end{tabular}

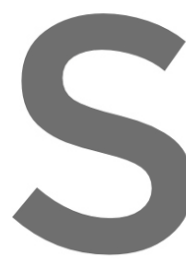

values are in good agreen discrepancies are noticed Those discrepancies might approximation of the aerol The laminar flow past a NACA0012 airfoil has al
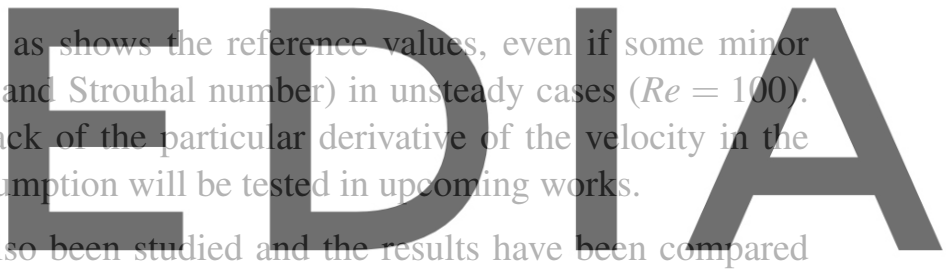

to data coming from a different simulation [27]. Figure 4 gives a representation of the streamlines

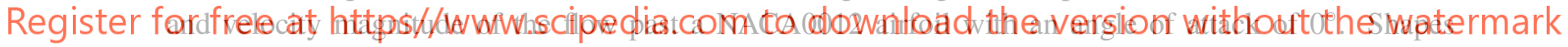
and recirculation length are really similar to the one presented in [27] (about $0.17 \mathrm{~m}$ in both cases). Concerning aerodynamic coefficients, we gathered the steady regime results obtained for angles of attack
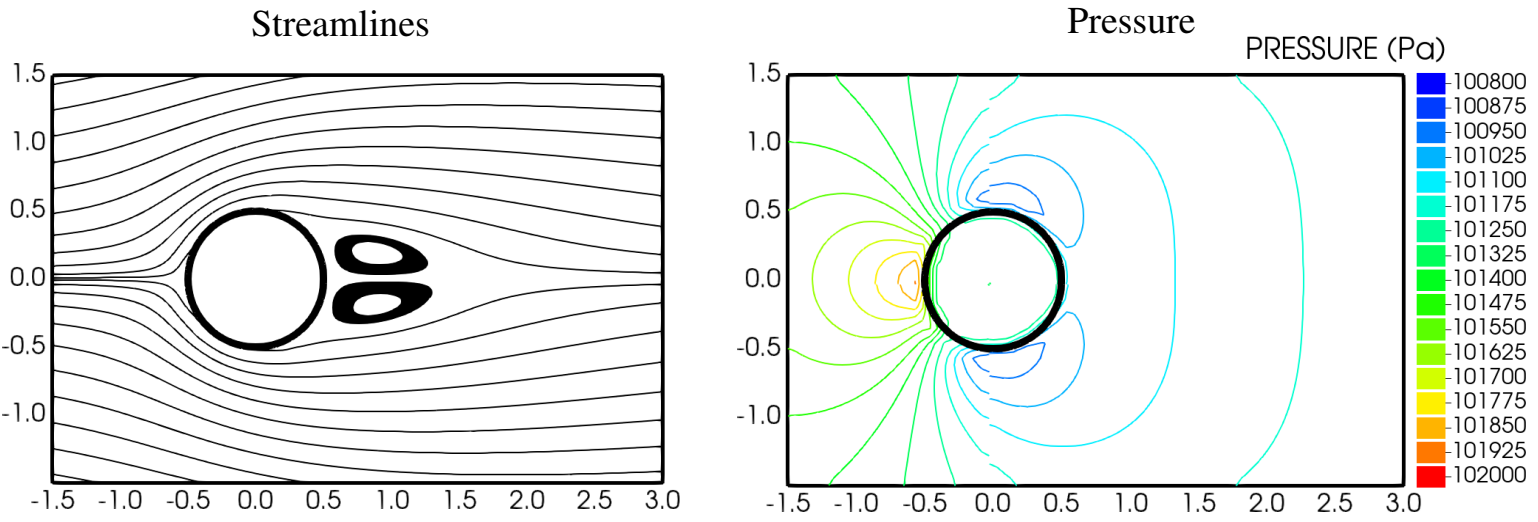

Figure 3: Streamlines and isobars of the steady flow around a static cylinder $(R e=20)$ 

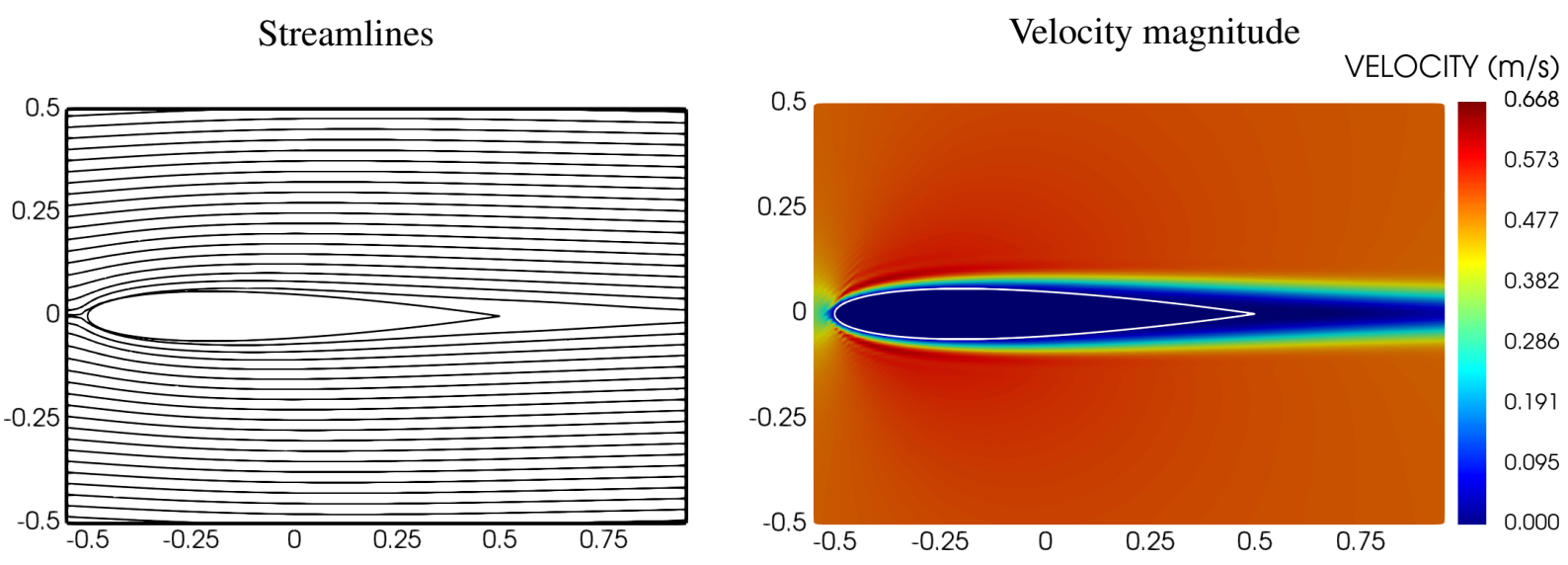

Figure 4: Computed streamlines and velocity magnitude map in the case of the flow past a NACA0012 airfoil with an angle of attack of $0^{\circ}$.

of 0 and $1^{\circ}$ in Table 5. Taking into account that an angle difference of $1^{\circ}$ can not be exactly reproduced using our immersed boundary geometrical model, we conclude that the aerodynamic coefficients are in a good agreement. However, for cases involving an angle of attack superior to $1^{\circ}$, our method was not able to recover steady state solutions so only the mean values of the aerodynamic coefficients are shown in Table 5. This can be induced by several phenomena such as:
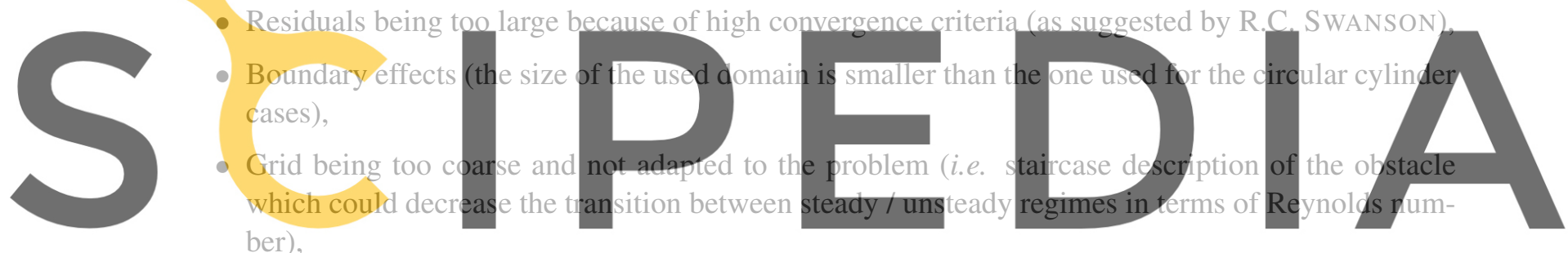

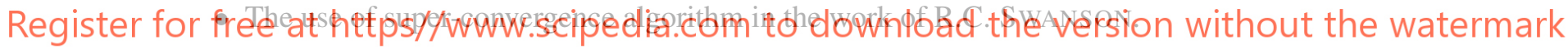

\section{CONCLUSION AND PERSPECTIVES}

In this document, the main theoretical aspects of the Penalized Direct Forcing method (an IBM whose characteristics inherit from both penalty and direct forcing methods) are briefly explained: it involves solving the Navier-Stokes equations using a fractional-step algorithm and an additional source term representative of the modeled obstacles (some kind of backmoving force). It was initially developed and tested in the context of a space-time discretization based on a finite difference scheme. Here, we specify and test the method using a Galerkin finite element discretization with a lumping of the mass matrix. Moreover, an enhancement of the method is also proposed : it consists in a directional interpolation of the velocity in the vicinity of the obstacles. Validation test cases are presented, as well as numerical results. The steady laminar Poiseuille and Taylor-Couette flows, for which analytical solutions are known, allowed us to carry out numerical convergence studies, with respect to the penalty parameter and the grid step. It is worth noting that the new linear interpolation technique reduces the difference between the computation results and the analytical solution while increasing the spatial order of convergence (almost 
reaching two for the velocity in $\mathcal{L}^{2}$ norm). Another validation case presented in this document is the laminar flow around a circular cylinder. It is declined in four configurations: steady regime with static cylinder, steady regime with rotating cylinder, unsteady regime with static cylinder and unsteady regime with rotating cylinder. Even if no analytical solution is available for the flow around a cylinder, a mesh convergence study is presented (the numerical results computed with the finest grid are considered as a reference) and shows, once again, that the interpolation technique increases the spatial order of the method. The results obtained with our approach is also compared to experimental data and other simulations via quantities such as aerodynamic coefficients and the Strouhal number on the circular cylinder test case and a laminar flow past a NACA0012 airfoil test case. Globally, the values obtained are in good agreement with the literature which provides valuable validation data.

In the near future, two developments are considered. The first one consists in interpolating the normal component of the pressure gradient in the vicinity of the immersed boundaries. Indeed, the velocity is interpolated using the presented methodology but not the pressure gradient (nor pressure corrector). Yet, the pressure gradient appears in the Navier-Stokes prediction equation. An idea to deal with this issue could be to interpolate the normal pressure gradient or corrector (as we consider $\nabla p^{n+1} \cdot \mathbf{n}=0$ as immersed boundary condition for the pressure) using the same methodology as the one used of the velocity. The second one involves turbulence modeling: the idea is to extend the interpolation process to turbulent wall laws (i.e. power wall law [28]). Thus, we will be able to carry out turbulent simulations of the flow limiter involving well-established turbulent models as the wall laws, RANS and LES models.
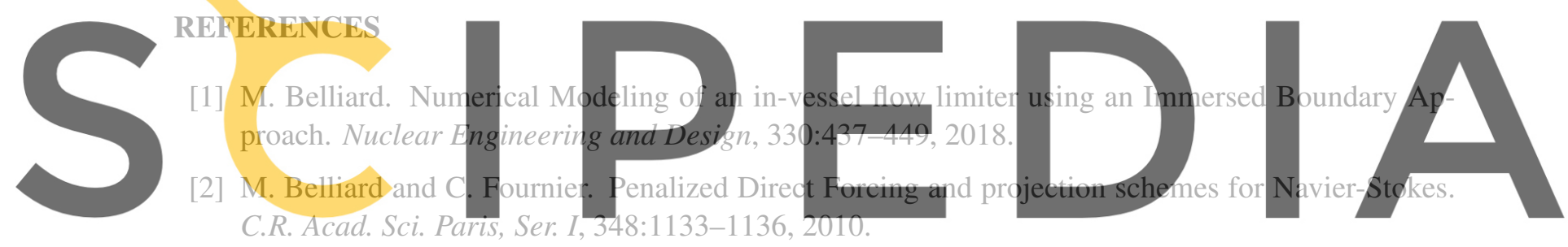

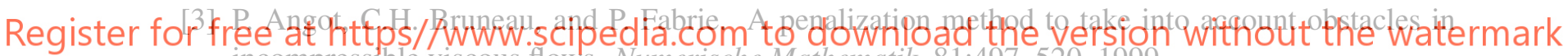
incompressible viscous flows. Numerische Mathematik, 81:497-520, 1999.

[4] C.S. Peskin. Flow Patterns around heart vavles: A digital computer method for solving the equations of motion. PhD thesis, Albert Einstein College of Medecine, 1972.

[5] D. L. Brown, R. Cortez, and M. L. Minion. Accurate projection methods for the incompressible Navier-Stokes equations. Journal of Computational Physics, 168:464-499, 2001.

[6] J. Mohd-Yusof. Combined Immersed-Boundary/B-spline methods for simulations of flow in complex geometries. Annual Research Briefs, pages 317-327, 1997.

[7] C.S. Peskin. The immersed boundary method. Acta Numerica, 11:479-517, 2002.

[8] R.D. Guy and D.A. Hertenstgine. On the accuracy of direct forcing immersed boundary methods with projection methods. Journal of Computational Physics, 229:2479-2496, 2010.

[9] V. Girault and P.A. Raviart. Finite Element Methods for Navier-Stokes Equations. Springer-Verlag, 1986.

[10] M. Grandotto and P. Obry. Calcul des coulements diphasiques dans les échangeurs par une méthode 
aux éléments finis. Revue européenne des éléments finis, 5:53-74, 1996.

[11] R. L. T. Bevan and P. Nithiarasu. Accelerating incompressible flow calculations using a quasiimplicit scheme: local and dual time stepping approaches. Computational Mechanics, 50:687-693, 2012.

[12] C. Intrö̈ni, M. Belliard, and C. Fournier. A second order penalized direct forcing for hybrid Cartesian/immersed boundary flow simulations. Computers \& Fluids, 90:21-41, February 2014.

[13] T. Ye, R. Mittal, H.S. Udaykumar, and W. Shyy. An accurate cartesian grid method for viscous incompressible flows with complex immersed boundaries. Journal of Computational Physics, 156(2):209 - 240, 1999.

[14] Jung-Il Choi, Roshan C. Oberoi, Jack R. Edwards, and Jacky A. Rosati. An immersed boundary method for complex incompressible flows. Journal of Computational Physics, 224(2):757 - 784, 2007.

[15] Kunihiko Taira and Tim Colonius. The immersed boundary method: A projection approach. Journal of Computational Physics, 225(2):2118 - 2137, 2007.

[16] Mark N. Linnick and Hermann F. Fasel. A high-order immersed interface method for simulating unsteady incompressible flows on irregular domains. Journal of Computational Physics, 204(1):157 $-192,2005$.

[17] Bengt Fornberg. A numerical study of steady viscous flow past a circular cylinder. Journal of Fluid Mechanics, 98(4):819-855, 1980.

[18] D. J. Tritton. Experiments on the flow past a circular cylinder at low reynolds numbers. Journal of Fluid Mechanics, 6(4):547-567, 1959.

[19] Meng-Hsuan Chung. Cartesian cut cell approach for simulating incompressible flows with rigid bodies of arbitrary shape. Computers \& Fluids, 35(6):607 - 623, 2006.

[20] D. Stojkovi, M. Breuer, and F. Durst. Effect of high rotation rates on the laminar flow around a circular cylinder. Physics of Fluids, 14(9):3160-3178, 2002.

[21] D.B. Ingham and T. Tang. A numerical investigation into the steady flow past a rotating circular cylinder at low and intermediate reynolds numbers. Journal of Computational Physics, 87(1):91 107, 1990.

[22] H.M. Badr, S.C.R. Dennis, and P.J.S. Young. Steady and unsteady flow past a rotating circular cylinder at low reynolds numbers. Computers \& Fluids, 17(4):579-609, 1989.

[23] P.H. Chiu, R.K. Lin, and Tony W.H. Sheu. A differentially interpolated direct forcing immersed boundary method for predicting incompressible navier-stokes equations in time-varying complex geometries. Journal of Computational Physics, 229(12):4476 - 4500, 2010.

[24] Yoann Cheny and Olivier Botella. The 1s-stag method: A new immersed boundary/level-set method for the computation of incompressible viscous flows in complex moving geometries with good conservation properties. Journal of Computational Physics, 229(4):1043 - 1076, 2010.

[25] C. Ji, A. Munjiza, and J.J.R. Williams. A novel iterative direct-forcing immersed boundary method and its finite volume applications. Journal of Computational Physics, 231(4):1797 - 1821, 2012. 
[26] S. Kang, H. Choi, and S. Lee. Laminar flow past a rotating circular cylinder. Physics of Fluids, 11(11):3312-3321, 1999.

[27] R.C. Swanson and S. Langer. Steady-state laminar flow solutions for NACA 0012 airfoil. Computers and Fluids, 126:102-128, 2016.

[28] S. Wilhelm, J. Jacob, and P. Sagaut. An explicit power-law-based wall model for lattice boltzmann method-reynolds-averaged numerical simulations of the flow around airfoils. Physics of Fluids, 30:065111, 2018. 